LBL-18063

SSC MAG-12

THE GENERALIZATION OF A CIRCULAR BUUUNDARY CUNOITION

IN THE PROGRAM POISSON TO INCLUDE NO SYMMETRY AND

AXIS-SYMMETRY OF REVOLUTION*

S. Caspi, M. Helm, and L.J. Laslett

Lawrence Berkeley Laboratory

University of California

Berkeley, California 94720

July 1984

* This work was supported by the Director, Office of Energy Research, Uffice of High Energy and Nuclear Physics, High Energy Physics Division, U.S. Dept. of Energy, under Contract No. DE-ACO3-765F00098. 
LBL-18063

SSC MAG-12

July 5,1984

\author{
THE GENERALIZATION OF A CIRCULAR BOUNDARY CONDITION IN \\ THE PROGRAM POISSON TO INCLUDE NO SYMMETRY AND \\ AXIS-SYMMETRY OF REVULUTION* \\ S. Caspi, M. Helm, and L.J. Laslett \\ Lawrence Berkeley Laboratory \\ University of California \\ Berkeley, California 94720
}

Introduction

We have previously reported on the incorporation of a circular boundary condition into the program POISSON for two-dimensional problems ("Incorporation of a Circular Boundary Condition into the Program POISSON", S. Caspi, M. Helm, and L.J. Laslett, LBID-887, SSC MAG Note-5, February 13, 1984). The least square method has now been generalized to accept any suitable set of orthogonal functions which can describe the vector potential function outside a circular boundary so located that no external sources are present. We have proceeded to incorporate the boundary condition into cartesian problems which involve no symmetry, and into axis-symetry cylindrical problems that may have left-right symmetry, antisymmetry or no syinmetry.

* This work was supported by the Director, Office of Energy Research, Office of High Energy and Nuclear Physics, High Energy Physics Division, U.S. Dept. of Energy, under Contract No. DE-ACO3-76SF00098. 
Analysis

Consider the case where a circular arc of radius $r=R-H \quad d i-$

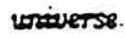
vides space into two regions (Fig. 1), an inner one which includes all current sources and magnetic iron, and an outer one which is in free space (hereafter referred to as the "universe"). Since the free space region is infinite we shall arbitrarily limit it by a secondary circular are of radius $r=R$. Both circular arcs are an assembly of connecting mesh

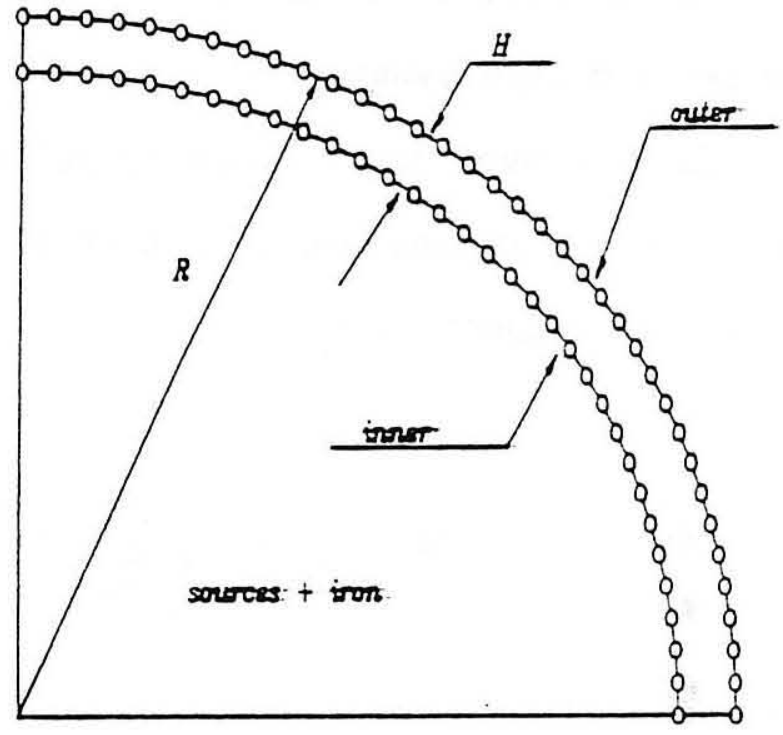

Fig. 1

points such as the one generated by the program LATTICE. If we know the vector potential for each mesh point on $r=R-H$ (e.g. calculated by POISSON), we would like to find the vector potential at each mesh point on $r=R$, so that such values may be employed as provisional boundary values in a subsequent relaxation pass through the entire mesh. This is expressed as:

$$
A_{k}^{\text {outer }}=\sum_{n=1}^{N} E_{k n} A_{n}^{\text {inner }}
$$

$A$ is the vector potential and $E$ is a working matrix, and the summation is over the entire mesh points of the inner arc.

In the free space region the vector potential can be expressed as a sum of harmonic terms, each employing powers of $1 / r$. 


$$
A_{i}=\sum_{\ell=1}^{\infty} r^{-a_{\ell}} D_{\ell} F_{\ell}\left(\theta_{i}\right)
$$

The vector potential $A$ of mesh point $i$ on the circular arc $r$ is expressed in terms of a series of functions $F_{2}(\theta)$, their coefficients $D_{2}$ and the problem type symmetry $a_{\ell}$.

Summing over the $N$ boundary points on the radius $r$, the difference between the calculated vector potential values and the relaxed ones is ininimized with respect to $D_{\ell}$.

Min: $\quad 1 / 2 \sum_{i=1}^{N} w_{i}\left(\sum_{\ell=1}^{m} r^{-a} \ell o_{\ell} F_{\ell}\left(\theta_{i}\right)-A_{i}\right)^{2}$

The number of harmonic terms has been reduced to $\mathrm{m}$ and the weight factors $W_{i}$ have been introduced to take care of an uneven distribution of mesh points along the boundary.

Following the minimization process we arrive at:

$$
\sum_{j=1}^{m} M_{i j} D_{j} r^{-\alpha} \ell=v_{i}
$$

where:

$$
\left.\begin{array}{rl}
M_{i j} & =\sum_{n=1}^{N} w_{n} F_{i}\left(\theta_{n}\right) F_{j}\left(\theta_{n}\right) \\
v_{i} & =\sum_{n=1}^{N} w_{n} F_{i}\left(\theta_{n}\right) A_{n}
\end{array}\right\} i, j=1,2,3 \ldots m
$$

Solving for $D_{j}$ on the inner arc $r=R-H$ we get

$$
o_{j}=\sum_{i=1}^{m}(R-H)^{\alpha_{j}}\left(M^{-1}\right)_{j i} v_{i}^{i n n e r}
$$


Using Eq. (2) on the outer arc $r=R$ and substituting the expressions for $D_{j}$ and $V_{i}$ we arrive at

$$
A_{k}^{\text {outer }}=\sum_{j=1}^{m}\left(\frac{R-H}{R}\right)^{a_{j}} F_{j}\left(\theta_{k}\right) \sum_{i=1}^{m}\left(M^{-1}\right)_{j i} \sum_{n=1}^{N} W_{n} F_{i}\left(\theta_{n}\right) A_{n}^{\text {inner }}
$$

Employing the working matrix $E_{x n}$, relation (6) is rewritten as:

$$
A_{k}^{\text {outer }}=\sum_{n=1}^{N} E_{k n} A_{n}^{\text {inner }}
$$

where

$$
\varepsilon_{k n}=\sum_{i=1}^{m} \sum_{j=1}^{m}\left(\frac{R-H}{R}\right)^{a j} w_{n}\left(M^{-1}\right)_{j i} F_{j}\left(\theta_{k}\right) F_{i}\left(\theta_{n}\right)
$$

Two Dimensional Case with Plane-Polar Cooroinates

The harmonic functions $F_{\ell}(\theta)$ are a combination of the trigonometric functions SIN and COS. It is, however, convenient to express them in the following way

$$
F_{l}(\theta)=\cos \left(a_{\ell} \theta-\beta_{l} \frac{\pi}{2}\right)
$$

The explicit functions are listed in the table below. 
$\ell \quad a \quad B_{\ell} \quad F_{\ell}(\theta) \quad$ Function

\begin{tabular}{|c|c|c|c|c|c|}
\hline 1 & 0 & 0 & $F_{1}$ & 1 & $\alpha_{2}=\frac{?}{2}$ integer division \\
\hline 2 & 1. & 1 & $F_{2}$ & $\sin \theta$ & \\
\hline 3 & 1 & 0 & $F_{3}$ & $\cos \theta$ & $B_{l}=\frac{\ell}{2}-\frac{\dot{i}-1}{2}$ integer \\
\hline 4 & 2 & 1 & $\mathrm{~F}_{4}$ & $\sin 2 \theta$ & division \\
\hline 5 & 2 & 0 & $F_{5}$ & $\cos 2 \theta$ & \\
\hline 6 & 3 & 1 & $F_{6}$ & $\sin 3 \theta$ & \\
\hline 7 & 3 & 0 & $F_{7}$ & $\cos 3 \theta$ & \\
\hline : & : & : & $\vdots$ & $\vdots$ & \\
\hline$\ell-1$ & $a_{b-1}$ & ${ }^{B} L-1$ & $F_{b-1}$ & $\sin \left(a_{2-1}\right.$ & $\theta)$ \\
\hline \& & $a_{2}$ & $B_{\ell}$ & $F_{\ell}$ & $\cos \left(\alpha_{2} \theta\right)$ & \\
\hline
\end{tabular}

Examples

Regular dipole:

The terms used to describe the vector potential of a regular dipole are:

$2=3,7,11, \ldots \ldots .4 k-1 ; k=1,2,3,4 \ldots$

$a_{2}=\frac{4 k-1}{2}$ integer division $\longrightarrow a_{k}=2 k-1$

$B_{2}=\frac{4 k-1}{2}-\frac{4 k-2}{2}$ integer division $\rightarrow B_{k}=0$

Regular quadrupole: $\ell=5,13,21, \ldots . .8 k-3 ; k=1,2,3,4 \ldots$.

$$
\begin{aligned}
& a_{\ell}=\frac{8 k-3}{2} \text { integer division } \rightarrow \alpha_{k}=4 k-2 \\
& { }_{B_{L}}=\frac{8 k-3}{2}-\frac{8 k-4}{2} \text { integer division } \rightarrow{ }_{k}=0
\end{aligned}
$$


Midplane symmetry: $\ell=1,3,5, \ldots .2 \mathrm{k}-1 ; k=1,2,3,4 \ldots$

$$
\begin{aligned}
& a_{\ell}=\frac{2 k-1}{2} \text { integer division } \rightarrow a_{k}=k-1 \\
& B_{\ell}=\frac{2 k-1}{2}-\frac{2 k-2}{2} \text { integer division } \rightarrow B_{k}=0
\end{aligned}
$$

No-s ymmetry: $\ell=1,2,3,4, \ldots \ldots k$;

$$
\begin{aligned}
& \alpha_{\ell}=\frac{k}{2} \text { integer division } \rightarrow \alpha_{k}=k-1 \\
& { }^{B_{\ell}}=\frac{k}{2}-\frac{k-1}{2} \text { integer division } \rightarrow B_{k}= \begin{cases}0 & k=1,3,5 \\
1 & k=2,4,6\end{cases}
\end{aligned}
$$

Ways of generating the $\alpha_{x}$ have been outlined in report LBID-847 ("The Vector Potential of Multiple Current Lines", S. Caspi, SSC-MAG-9, February 27,1984$)$ and are summarized in the table below.

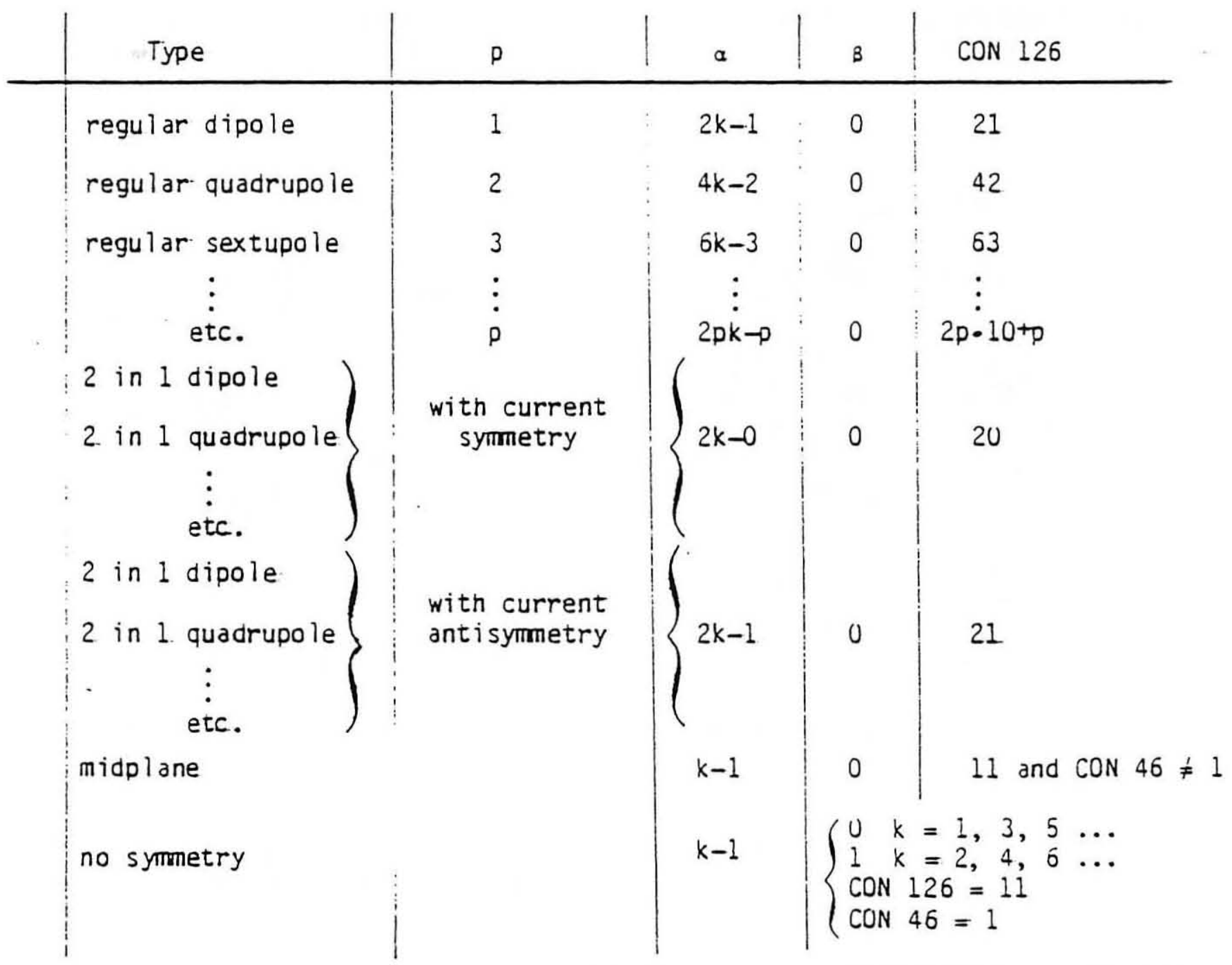


Figures 2, 3, and 4 are flux line plots which demonstrate the method introduced here for the use of boundary conditions. All cases are for Cartesian problems.
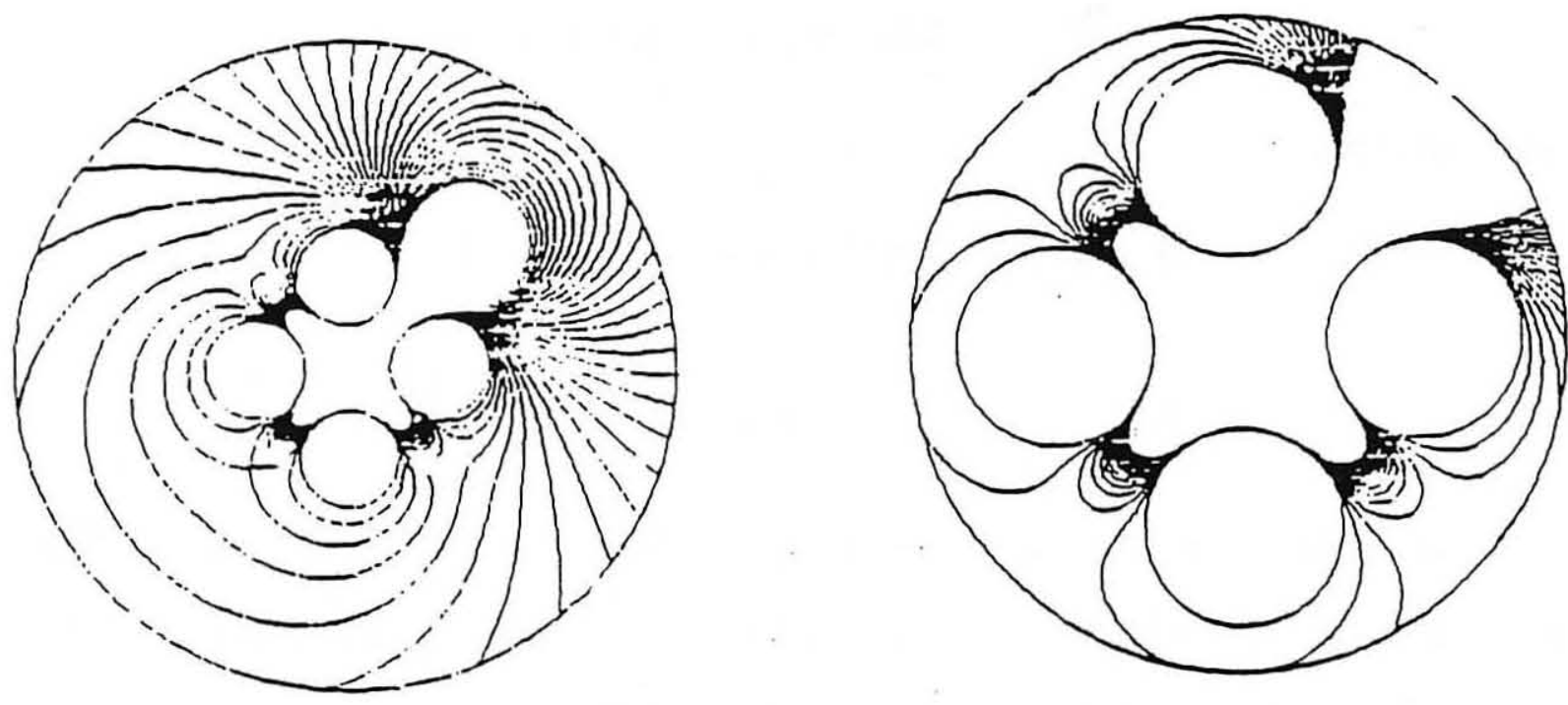

(a) Boundary at $R=16.0$ Source at $45^{\circ}$

(b) Boundary at $R=8.0$ Source at $45^{\circ}$

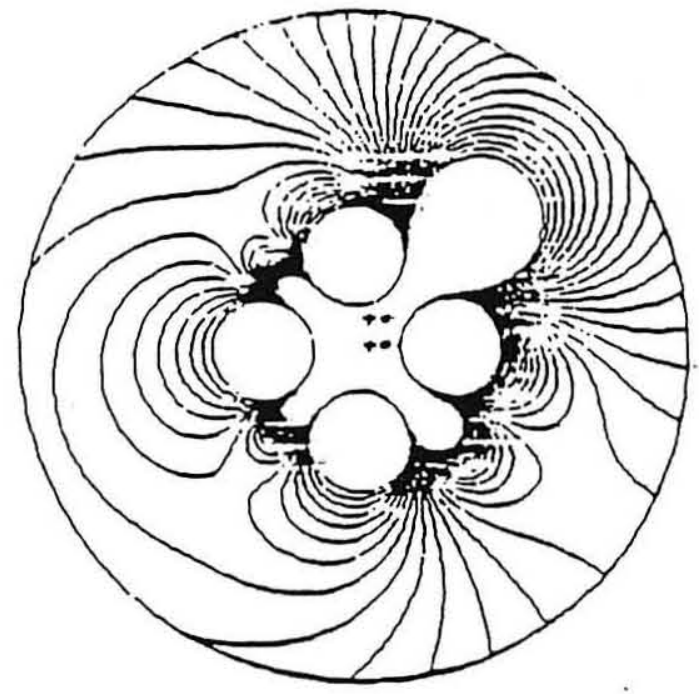

(c) No symmetry in current source.

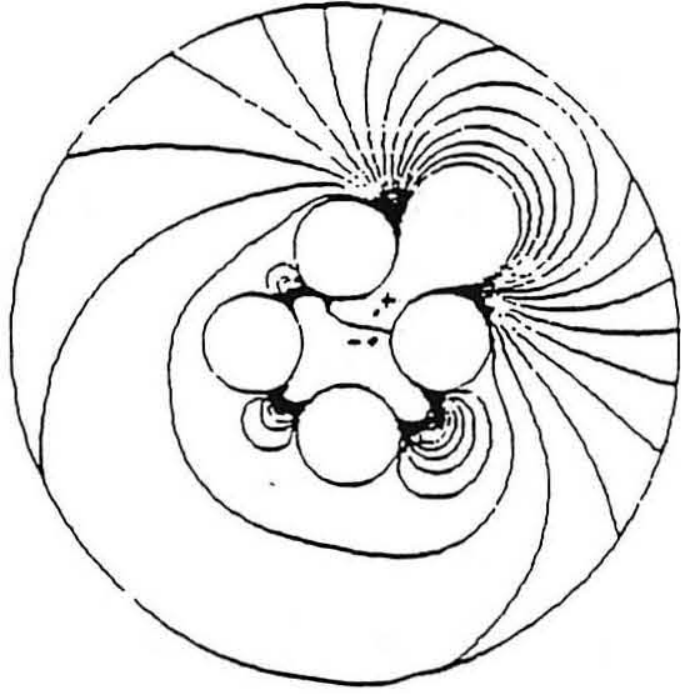

(d) No symmetry in current flow.

Fig. 2 Flux lines are plotted for cases where the use of nosymmetry in the boundary condition has been applied. $\left(\operatorname{CON} 46=1, \alpha_{x}=11\right)$. 


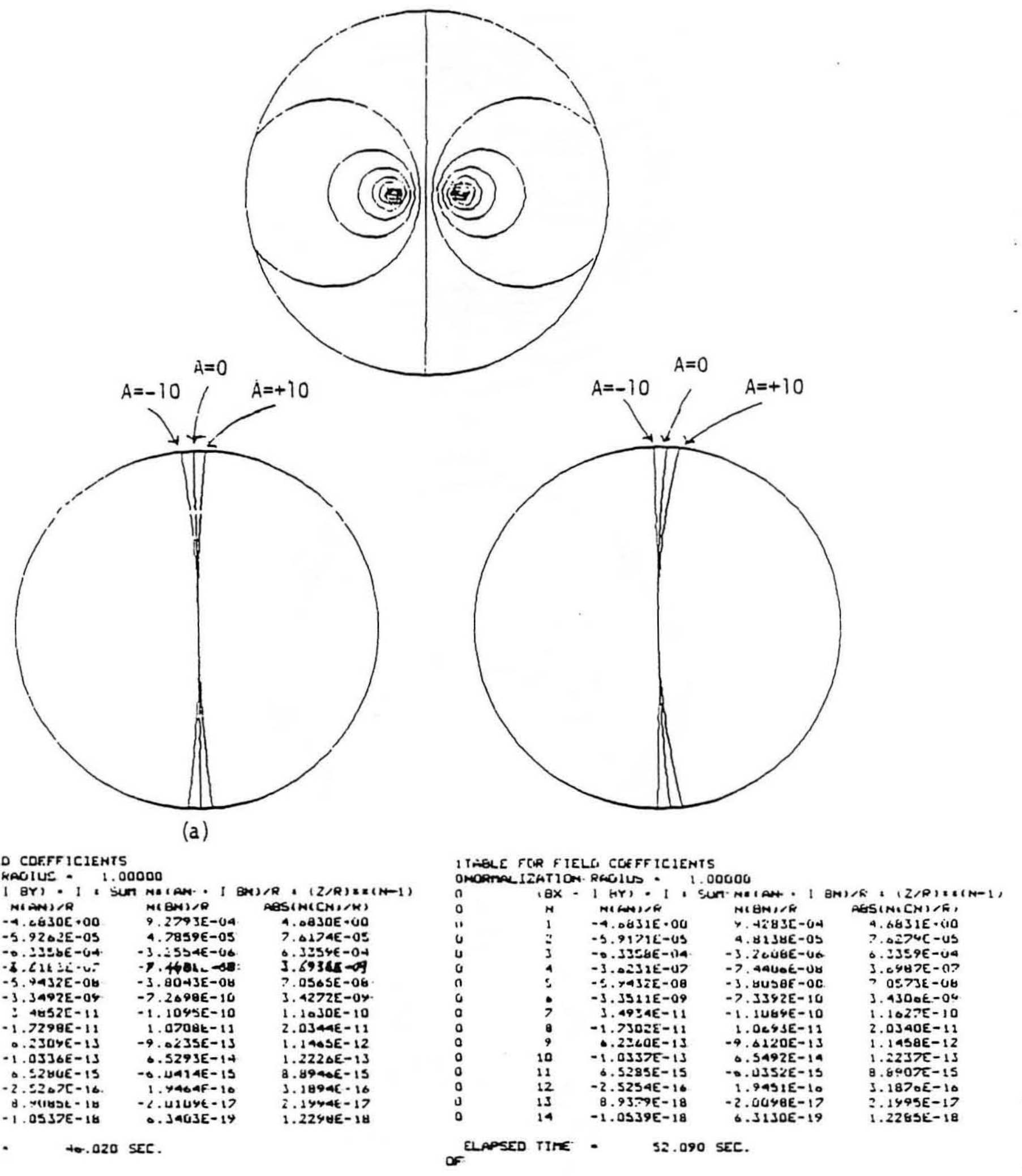

Fig. 3 Flux lines for a single current linc dipole. The boundary conditions are such that all oossible harmonics are alTowed. The fine symmetry is preserved if the vector potential at $(0,0)$ is set to 0 (case a). Both cases result in the same field harmonics. 

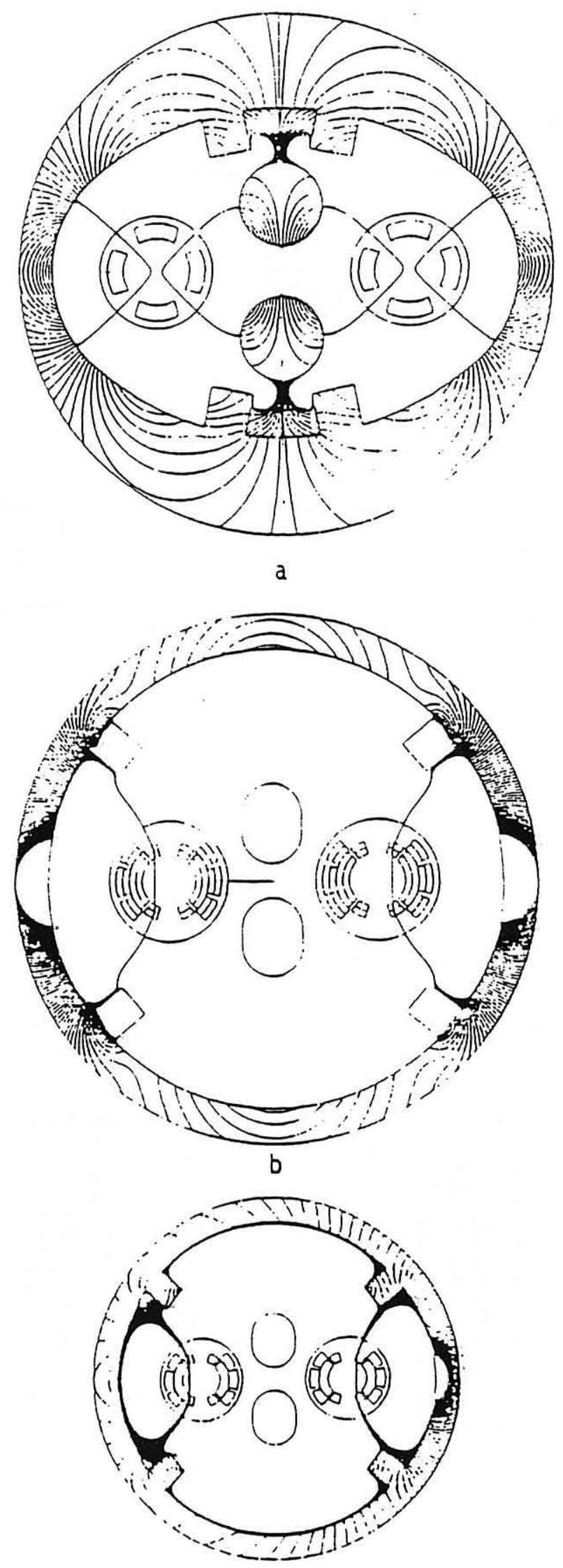

C

Fig. 4 Flux lines leaking from the iron to the "universe" are plotted for (a) 2 -in-1 quadrupole $a_{k}=2 k-1$; (b) SSC $2-i n-1$ dipole with even current loading, $\alpha_{k}=2 k-0$; (c) SSC 2 -in-1 dipole with uneyen loading $a_{k}=k-1$. Case $a$ and $b$ were solved in a single quadrant, 
Axis-Symmetry Problems with Polar Coordinates

Here we consider cases which obey symmetry with respect to revolution around the $Z$ axis. The component $A_{\phi}$ of a vector potential $\vec{A}=A(r, \theta) \hat{e}_{\phi}$ in a spherical coordinate system may employ terms of the form

$$
r^{n} p_{n}^{1}(u) \text { and } \frac{1}{r^{n+1}} p_{n}^{1}(u)
$$

in addition, in principle, to terms of the form $\frac{a}{r}+b$.

$p_{n}^{1}(u)$ are the associated Legendre functions.

(See for example "Mathematical Methods for Physicists", G. Arfken, pp. 558-567).

Here $r=\left(0^{2}+z^{2}\right)^{1 / 2}, 0=r \sin \theta, z=r \cos \theta$, and $u=\cos \theta$ with $\theta$ denoting the angle from the pole (co-latitude).

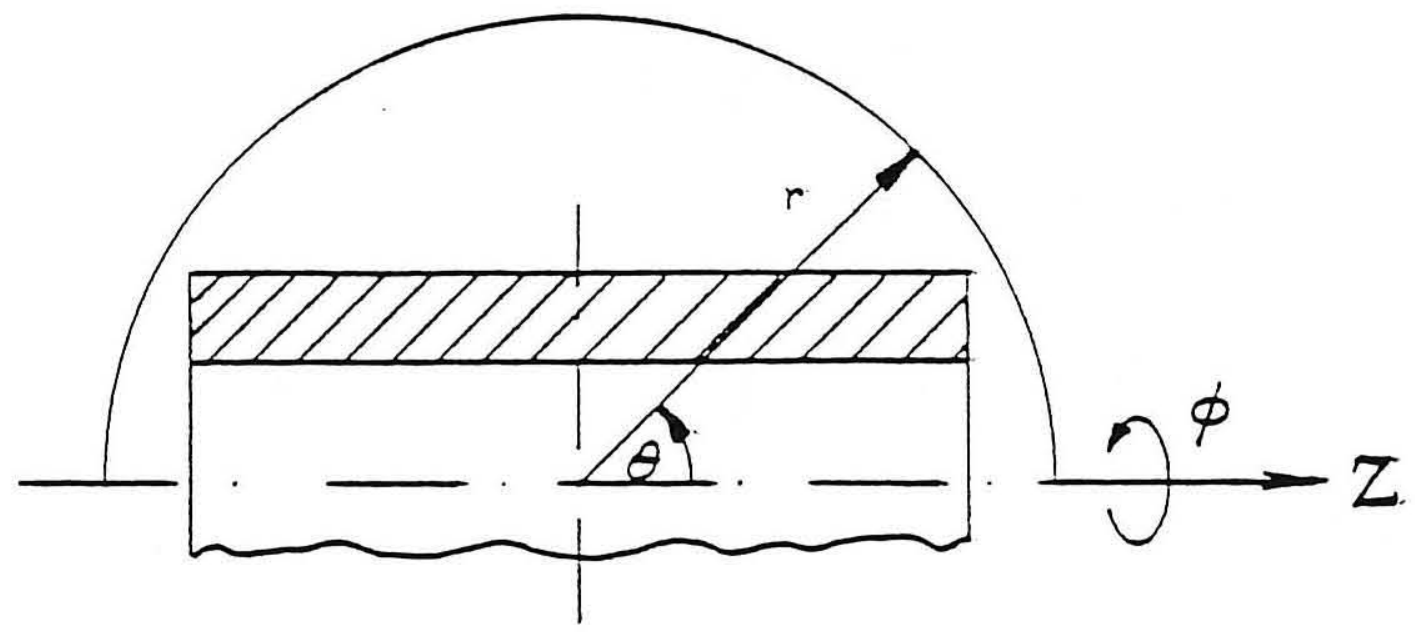

In a region $(r>R)$ deyoid of external sources we may expect that $A_{\phi}$ can be represented exclusively in terms of the form

$$
\frac{1}{r^{2+1}} p_{2}^{1}(\cos \theta) \quad[l=1,2, \ldots]
$$


We proceed to somewhat normalize the associated Legendre function. This can sometimes improve the inversion of $M_{i j}$ and express the vector potential in the following form

$$
A_{\phi}(r, \theta)=\sum_{\ell=1}^{\infty} r^{-(\ell+1)} D_{\ell} \cdot\left(\frac{P_{\ell}^{1}(\cos \theta)}{\ell}\right)
$$

We recognize the fact that our normalization factor $\ell$ is different from the conventional one $\frac{\ell(\ell+1)}{\ell+\frac{1}{2}}$.

In a cylindrical geometry the flux lines are represented by the product o. $A_{\phi}$ where $A_{\phi}$ is the vector potential. The program POISSON is written in such a way that this product is the one which is being relaxed. We. therefore proceed and redefine:

$$
A_{\phi}^{*}=\rho \cdot A_{\phi} \quad ; \quad \rho=r \sin \theta
$$

Equation 8 is now written as:

$$
A_{\gamma}{ }^{\star}=\sum_{\ell=1}^{\infty} r^{-\ell} D_{\ell}\left(\frac{\sin \theta \cdot P_{\ell}^{1}(\cos \theta)}{\ell}\right)
$$

We define:

$$
F_{\ell}=\frac{\sin \theta \cdot p_{\alpha_{2}}^{1}(\cos \theta)}{\alpha_{2}} ; \quad \alpha_{2}=\ell \quad-1 \leq \cos \theta \leq 1
$$

Such Legendre associated functions may serve to describe a magnetic vectorpotential function in certain cases of cylindrical symmetry. We write down the explicit form of $F_{2}$ in the following table: 


\begin{tabular}{c|c|c|c|c}
$\ell$ & $a$ & $F$ & Function \\
\hline 1 & 1 & $F_{1}$ & $\sin \theta P_{1}{ }^{1}(\cos \theta)$ \\
2 & 2 & $F_{2}$ & $\frac{\sin \theta}{2} P_{2}{ }^{1}(\cos \theta)$ \\
3 & 3 & $F_{3}$ & $\frac{\sin \theta}{3} P_{3}{ }^{1}(\cos \theta)$ \\
2 & $\vdots$ & $\vdots$ & $\vdots$ \\
& 2 & $F_{2}$ & $\frac{\sin \theta}{2} P_{2}^{1}(\cos \theta)$
\end{tabular}

Symmetry with respect to equatorial plane: $\ell=1,3,5 \ldots 2 k-1 ; k=1,2,3 \ldots$ We note that if $A_{\phi}$ is even about $\theta=\pi / 2$ (current symmetry), only odd values of $\ell$ are required.

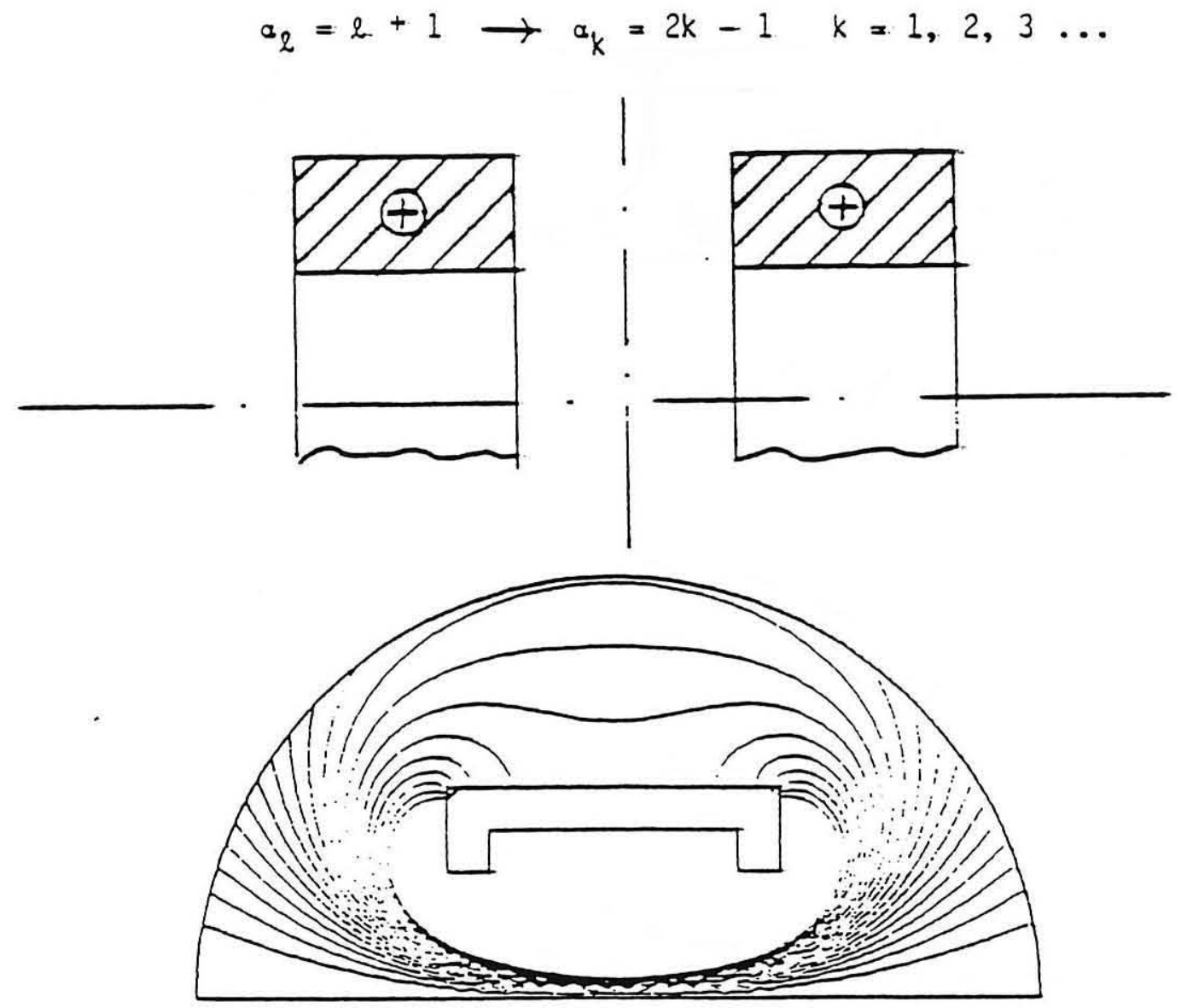

(a) $a_{x}=2 x-1 ; k=1,2,3 \ldots$ 
Antisymmetry with respect to equatorial plane: $\ell=2,4,6 \ldots 2 k ; k=1,2,3 \ldots$ If $A_{\phi}$ is odd about $\theta=\pi / 2$ (current antisymetry), only even values of $l$ are required.
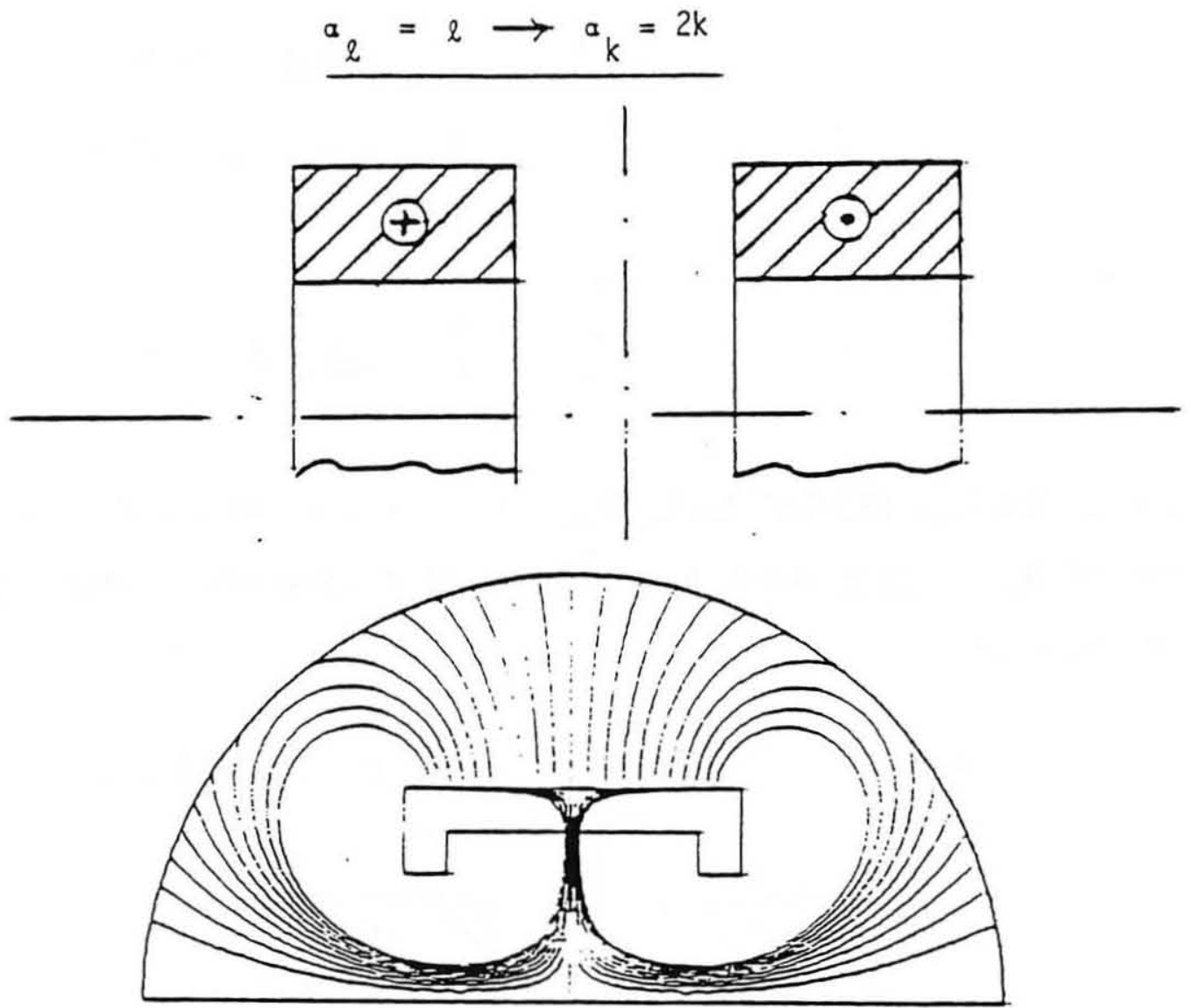

(b) $a_{k}=2 k$

No symmetry: $\quad l=1,2, \ldots k \rightarrow a_{k}=k$.

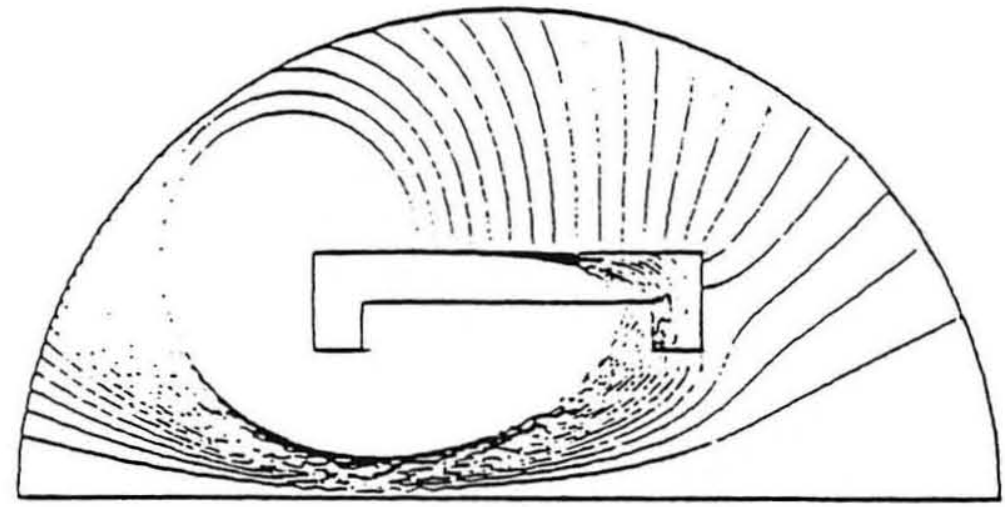

(c) $\alpha_{k}=k$ 
We summarize the results for POISSON in the following table (CON 126 is one of the POISSON constants).

\begin{tabular}{l|c|c|c}
$\begin{array}{c}\text { Type } \\
(\operatorname{CON} 19=1)\end{array}$ & $\alpha$ & $\operatorname{CON} 126$ & $\operatorname{CON} 46$ \\
\hline Symmetry & $2 k-1$ & 21 & $\left\{\begin{array}{l}1 \text { for 2 quadrants } \\
2 \text { for 1 quandrant } \\
\text { Antisymmetry }\end{array}\right.$ \\
No-Symmetry & $x$ & 20 & or midplane
\end{tabular}

\section{Recursion Relation}

The computations of the Legendre functions are based on the recursion relation

$$
(2-1) \&\left[\frac{P_{l}^{1}(u)}{L}\right]=(22-1)(2-1) u\left[\frac{P_{l-1}^{1}(u)}{2-1}\right]-2(2-2)\left[\frac{P_{l}^{1}-2}{2-2}\right]
$$

For those cases which require only odd or even tems we have a related recursion relation that relates Legendre functions whose degrees (subscripts) differ by two units (rather than by one unit, as above).

$$
\frac{P_{2}^{1}(u)}{2}=
$$

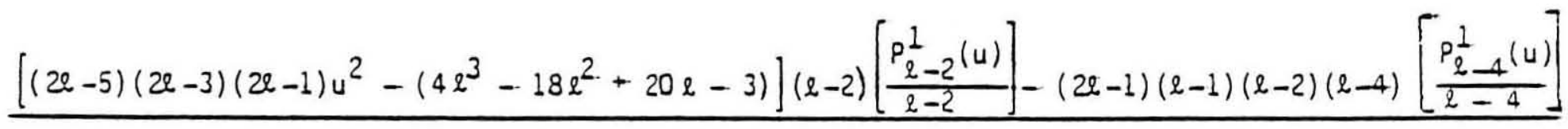

$$
\begin{aligned}
& (22-5)(2-1)(2-2) 2
\end{aligned}
$$

We have prepared a subroutine SALFN (together with an attacned program ASSOL, for testing this subroutine) intended to provide values of

$$
F_{2}=\frac{P_{2}^{1}(u)}{2}
$$


More on Symmetries

In the course of developing some general rules for the values of the harmonic terms $a_{k}$ we realized that in some cases the leading term in the vector potential series decays radially with a high power. (Again, we are only concerned with the potential in the region outside all sources). These cases involve problems with a large number of poles and a geometry that obey internal symmetry. The program POISSON, written to accommodate the present type of boundary condition, assumes however that pseudo termis do exist prior to the leading term and should drop out as the problem continues to relax. Since this type of problem presently is of only academic interest, we have. included such terms here without addressing them in the POISSON code itself.

We use the mathematical argument that the derivative of one multipole leads to the next higher multipole (e.g. in two dimensional space dipoles are second rank tensors).

\section{Cartesian Coordinates}

For this example we choose to expand the vector potential $A_{z}$ in the $x$ direction only ( $A_{z}$ is "symmetrical" in the sense that only cos terms are emp loyed)

$$
\begin{gathered}
A_{z}-\frac{1}{r^{a_{k}}} \cos \left(a_{k} \theta\right) \\
\frac{\partial A_{z}}{\partial x}=\left(\cos \theta \cdot \frac{\partial}{\partial r}-\sin \theta \frac{1}{r} \frac{\partial}{\partial \theta}\right) A_{z} \\
\frac{\partial A_{z}}{\partial x}=-\frac{a_{k}}{r^{a^{+}} 1} \cos \left[\left(a_{k}+1\right) \theta\right] \\
\frac{\partial^{2} A_{z}}{\partial x^{2}}=\frac{a_{k}\left(a_{k}+1\right)}{r^{a^{+}}} \cos \left[\left(a_{k}+2\right) \theta\right] \quad \text { etc. } \ldots
\end{gathered}
$$


If we apply the rule to a single dipole $a_{k}=2 k-1$ we get

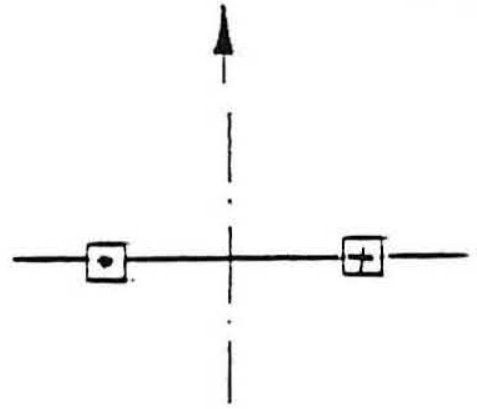

ant isymmetry

$\frac{1}{r^{2 k-1}} \cos [(2 k-1) \theta]$

$\frac{1}{r} \cos \theta, \ldots$

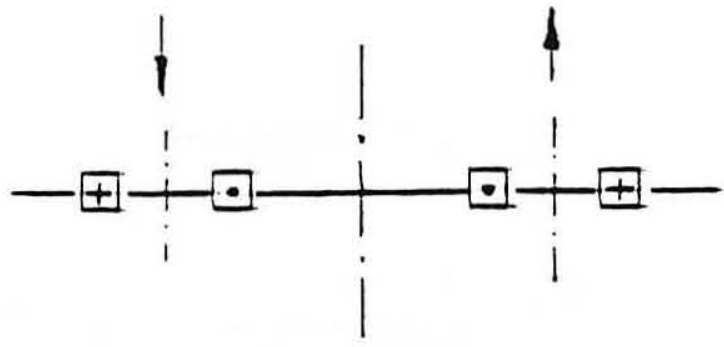

symmetry

$\frac{1}{r^{2 k}} \cos (2 k \theta)$

$\frac{1}{r^{2}} \cos 2 \theta, \cdots$

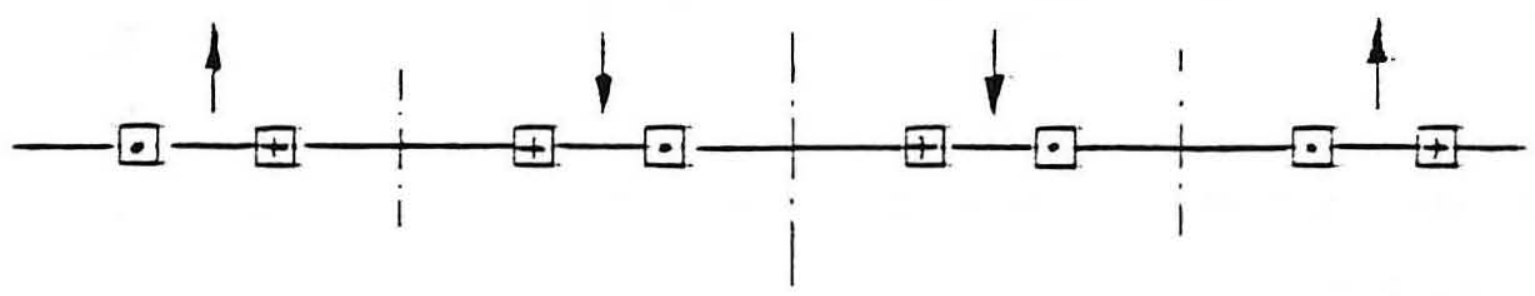

antisymetry

$$
\frac{1}{r^{2 k+1}} \cos [(2 k+1) \theta]
$$$$
\frac{1}{r^{3}} \cos 3 \theta, \ldots
$$

Finite-difference illustrations only

We note that the leading term in case (c) decays with the third power whereas the one in case (a) decays with the first one. However running case (c) on POISSON the first term would have been of the same order as in case (a) assuming, though, that as the problem continues to relax the coefficient for $(1 / r)$ will continue to decline letting the term $\left(1 / r^{3}\right)$ become dominant. The same process will be true running POISSON for cases with a larger number of poles where the leading term takes the form of $1 / r^{n}$ with $n>1$. 


\section{Polar Coordinates}

We differentiate the vector potential $A_{\phi}$ with respect to axial symmetry axis.

$$
\begin{aligned}
& A_{\phi}-\frac{1}{r^{a_{k}^{+}}+1} P_{a_{k}}^{1}(\cos \theta) \\
& \frac{\partial A_{\phi}}{\partial z}=\left(\cos \theta \cdot \frac{\partial}{\partial r}-\sin \theta \frac{1}{r} \frac{\partial}{\partial \theta}\right) A_{\phi} \\
& \frac{\partial A_{\phi}}{\partial z}=-\frac{a_{k}}{r^{a_{k}-2}} p_{a_{k}+1}^{1}(\cos \theta) \\
& \frac{\partial^{2} A_{\phi}}{\partial z^{2}}=\frac{a_{k}\left(a_{k}+1\right)}{r^{a_{k}+3}} p_{a_{k}+2}^{1}(\cos \theta) \text { etc. ... }
\end{aligned}
$$

Applying the rule to a solenoid with $a_{k}=2 k-1$ we can similarly develop groups of them with high decay power in their leading terms.

To differentiate the associated Legendre polynomials we make use of Eq. (8.5.4) and (8.5.3) in the book of Abramowitz and Stegun (p. 334 ) to obtain

$$
\begin{aligned}
\frac{d P_{n}^{1}(x)}{d x} & =\frac{(n+1) \times P_{n}^{1}(x)-n P_{n+1}^{1}(x)}{1-x^{2}} \\
x & =\cos \theta .
\end{aligned}
$$




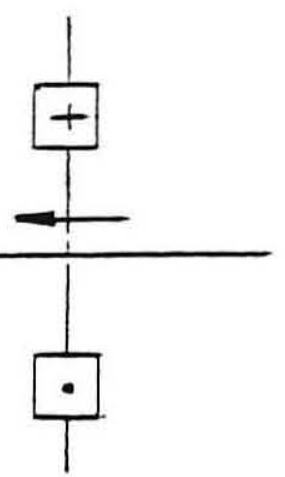

symmetry

$\frac{1}{r^{2 k}} p^{1} \frac{1}{2 k-1}(u)$

$\frac{1}{r^{2}} p_{1}^{1}, \ldots$

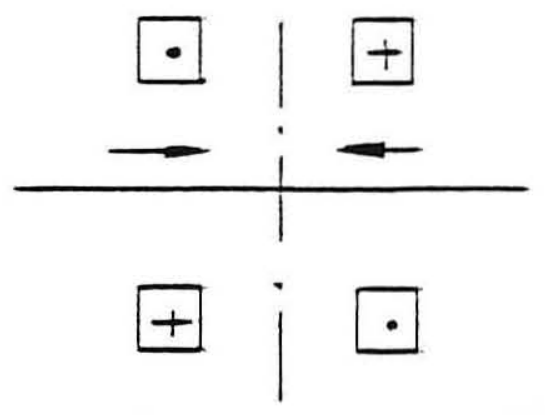

anti symmetry

$\frac{1}{r^{2 k+1}} p^{1} \frac{1}{2 k}(u)$

$\frac{1}{r^{3}} p_{2}^{1}, \ldots$

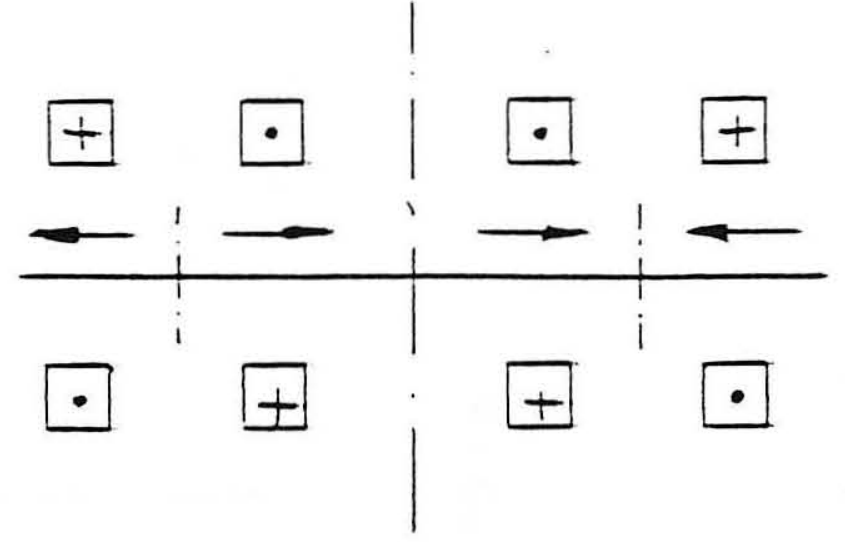

symmetry

$\frac{1}{r^{2 k+2}} P^{1} \frac{1}{2 k+1}(u)$

$\frac{1}{r^{4}} p_{3}^{1}, \ldots$

Examole

We have calculated the vector potential for a single current loop and compared the results with those from POISSON.

We express the vector potential of a single current loop placed at $z=0$ and $\rho=a=1$ as:

$$
\begin{aligned}
& A_{\phi}=\frac{1}{10 \rho} \sqrt{(a+o)^{2}+z^{2}}\left[\left(1+m_{1}\right) x-2 E\right] \\
& m_{1}=\frac{(a-o)^{2}+z^{2}}{(a+o)^{2}+z^{2}}
\end{aligned}
$$

$K$, $E$ are the elliptic integral of the first and second $k$ ind respectively.

For $I=1 \mathrm{~A}$ we have solved for the vector potential using POISSON and compared results for cases that use different boundary conditions. (Fig. 5). 


\section{The Vector Potential of a Single Loop}

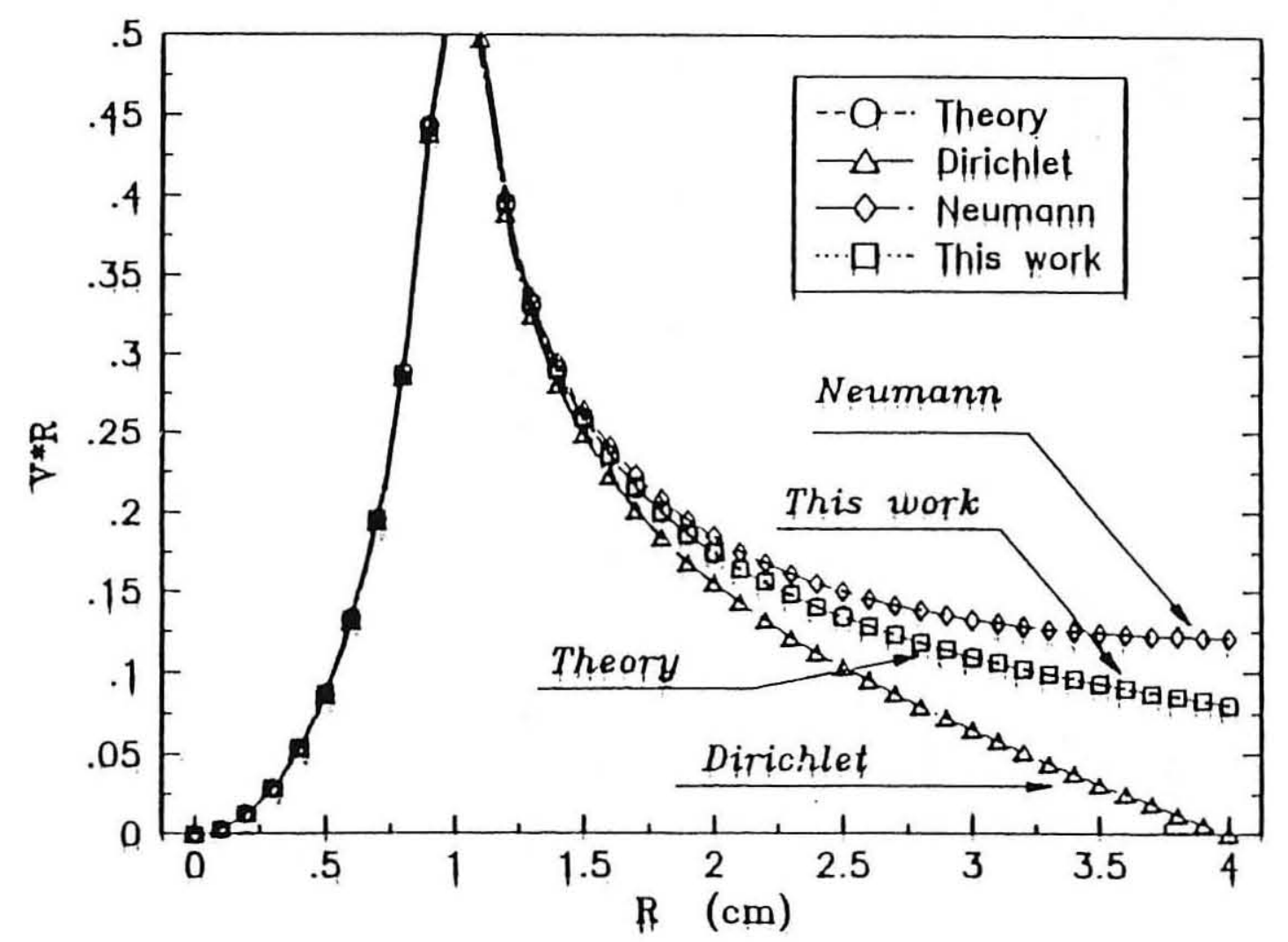

Fig. 5 The vector potential, along $r$ at $z=0$, of a single current loop carrying iA subjected to various boundary conditions. 